\title{
International Geomagnetic Reference Field—the tenth generation
}

\author{
Susan Macmillan ${ }^{1}$ and Stefan Maus $^{2}$ \\ ${ }^{1}$ British Geological Survey, Murchison House, West Mains Road, Edinburgh, EH9 3LA, U.K. \\ ${ }^{2}$ National Geophysical Data Center, E/GC 325 Broadway, Boulder, Colorado, 80305-3328, U.S.A.
}

(Received March 28, 2005; Accepted May 9, 2005)

\begin{abstract}
The International Geomagnetic Reference Field (IGRF) 10th Generation was adopted in 2004 by the International Association of Geomagnetism and Aeronomy (IAGA) Working Group V-MOD. It is the latest version of a standard mathematical description of the Earth's main magnetic field and is used widely in studies of the Earth's deep interior, its crust and its ionosphere and magnetosphere. This generation differs from the previous generation with the replacement of the secular-variation model for 2000.0-2005.0 with a main-field model at 2005.0 and a secular-variation model for 2005.0-2010.0. The IGRF is the product of a huge collaborative effort between magnetic field modellers and the institutes involved in collecting and disseminating magnetic field data from satellites and from observatories and surveys around the world. This paper lists the new coefficients and includes contour maps and pole positions.
\end{abstract}

Key words: Main field modelling, IGRF.

\section{Introduction}

The IGRF is an internationally agreed series of global spherical harmonic models of the Earth's magnetic field whose sources are mainly in the Earth's core. In sourcefree regions at the Earth's surface and above, the main field, with sources inside the Earth, is the negative gradient of a scalar potential $V$ which can be represented by a truncated series expansion

$$
\begin{aligned}
V(r, \theta, \lambda, t)= & R \sum_{n=1}^{n_{\max }}\left(\frac{R}{r}\right)^{n+1} \sum_{m=0}^{n}\left(g_{n}^{m}(t) \cos m \lambda\right. \\
& \left.+h_{n}^{m}(t) \sin m \lambda\right) P_{n}^{m}(\theta)
\end{aligned}
$$

where $r, \theta, \lambda$ are geocentric coordinates ( $r$ is the distance from the centre of the Earth, $\theta$ is the colatitude, i.e. $90^{\circ}$ latitude, and $\lambda$ is the longitude), $R$ is a reference radius $(6371.2 \mathrm{~km}) ; g_{n}^{m}(t)$ and $h_{n}^{m}(t)$ are the coefficients at time $t$ and $P_{n}^{m}(\theta)$ are the Schmidt semi-normalised associated Legendre functions of degree $n$ and order $m$. The coefficients are functions of time and for the IGRF they are assumed to vary at constant rates for five-year intervals. For more details on main-field modelling the reader is referred to Chapman and Bartels (1940) and Langel (1987).

The first generation of the IGRF was available in 1969, and this current revision is the 10th generation of the IGRF. For a history of the IGRF see Barton (1997). The new constituent models are a main-field model for 2005.0 and a secular-variation model for 2005.0-2010.0.

Copyright (c) The Society of Geomagnetism and Earth, Planetary and Space Sciences (SGEPSS); The Seismological Society of Japan; The Volcanological Society of Japan; The Geodetic Society of Japan; The Japanese Society for Planetary Sciences; TERRAPUB

\section{Development of New Constituent Models for IGRF-10}

The call for candidate main-field models at epoch 2005.0 to maximum degree 13 and for annual secular-variation models to maximum degree 8 for 2005.0-2010.0 went out in March 2004 from the chairman, Stefan Maus, of the IAGA Working Group V-MOD Task Force and in response, models were received in October 2004 from four teams. The teams are: Danish Space Research Institute (Denmark), Goddard Space Flight Center (USA) and Newcastle University (UK) - team A; National Geophysical Data Center (USA), GeoForschungZentrum (Germany)—-team B; British Geological Survey (UK) — team C; and Institute of Terrestrial Magnetism, Ionosphere and Radio Wave Propagation (Russia) - team D.

Whilst all teams have had access to similar datasets the most distinctive features of each of their contributions may be summarised as follows. Team A applied ionospheric corrections, team B applied diamagnetic plasma correction to CHAMP data and oceanic tidal corrections to all input data, team $\mathrm{C}$ used observatory hourly mean data and incorporated outputs from linear prediction filters to long-term annual mean data into their secular-variation model, and team D used natural orthogonal component analysis. Sections 1 and 2 of Maus et al. (2005a) give some more information about the candidate models submitted, but for detailed information the reader is referred to Olsen et al. (2005), Maus et al. (2005b), Lesur et al. (2005) and Golovkov et al. (2005) in this special issue of the journal Earth, Planets and Space.

A web page was set up and the models with associated descriptions were posted during October 2004 (www.ngdc.noaa.gov/IAGA/vmod/IGRF-10). In November 2004 various evaluations of the candidate models were posted, and these are summarised in Maus et al. (2005a), and the final decision on the selection and weighting of 
Table 1. Spherical harmonic (Gauss) coefficients for the IGRF main-field model at 2005.0 and secular-variation model for 2005.0-2010.0 in the 10th Generation IGRF.

\begin{tabular}{|c|c|c|c|c|}
\hline$g / h$ & $n$ & $m$ & $\begin{array}{c}\text { MF } 2005 \\
(\mathrm{nT})\end{array}$ & $\begin{array}{c}\text { SV 2005-2010 } \\
\text { (nT/year) }\end{array}$ \\
\hline$g$ & 1 & 0 & -29556.8 & 8.8 \\
\hline$g$ & 1 & 1 & -1671.8 & 10.8 \\
\hline$h$ & 1 & 1 & 5080.0 & -21.3 \\
\hline$g$ & 2 & 0 & -2340.5 & -15.0 \\
\hline$g$ & 2 & 1 & 3047.0 & -6.9 \\
\hline$h$ & 2 & 1 & -2594.9 & -23.3 \\
\hline$g$ & 2 & 2 & 1656.9 & -1.0 \\
\hline$h$ & 2 & 2 & -516.7 & -14.0 \\
\hline$g$ & 3 & 0 & 1335.7 & -0.3 \\
\hline$g$ & 3 & 1 & -2305.3 & -3.1 \\
\hline$h$ & 3 & 1 & -200.4 & 5.4 \\
\hline$g$ & 3 & 2 & 1246.8 & -0.9 \\
\hline$h$ & 3 & 2 & 269.3 & -6.5 \\
\hline$g$ & 3 & 3 & 674.4 & -6.8 \\
\hline$h$ & 3 & 3 & -524.5 & -2.0 \\
\hline$g$ & 4 & 0 & 919.8 & -2.5 \\
\hline$g$ & 4 & 1 & 798.2 & 2.8 \\
\hline$h$ & 4 & 1 & 281.4 & 2.0 \\
\hline$g$ & 4 & 2 & 211.5 & -7.1 \\
\hline$h$ & 4 & 2 & -225.8 & 1.8 \\
\hline$g$ & 4 & 3 & -379.5 & 5.9 \\
\hline$h$ & 4 & 3 & 145.7 & 5.6 \\
\hline$g$ & 4 & 4 & 100.2 & -3.2 \\
\hline$h$ & 4 & 4 & -304.7 & 0.0 \\
\hline$g$ & 5 & 0 & -227.6 & -2.6 \\
\hline$g$ & 5 & 1 & 354.4 & 0.4 \\
\hline$h$ & 5 & 1 & 42.7 & 0.1 \\
\hline$g$ & 5 & 2 & 208.8 & -3.0 \\
\hline$h$ & 5 & 2 & 179.8 & 1.8 \\
\hline$g$ & 5 & 3 & -136.6 & -1.2 \\
\hline$h$ & 5 & 3 & -123.0 & 2.0 \\
\hline$g$ & 5 & 4 & -168.3 & 0.2 \\
\hline$h$ & 5 & 4 & -19.5 & 4.5 \\
\hline$g$ & 5 & 5 & -14.1 & -0.6 \\
\hline$h$ & 5 & 5 & 103.6 & -1.0 \\
\hline$g$ & 6 & 0 & 72.9 & -0.8 \\
\hline$g$ & 6 & 1 & 69.6 & 0.2 \\
\hline$h$ & 6 & 1 & -20.2 & -0.4 \\
\hline$g$ & 6 & 2 & 76.6 & -0.2 \\
\hline$h$ & 6 & 2 & 54.7 & -1.9 \\
\hline$g$ & 6 & 3 & -151.1 & 2.1 \\
\hline$h$ & 6 & 3 & 63.7 & -0.4 \\
\hline$g$ & 6 & 4 & -15.0 & -2.1 \\
\hline$h$ & 6 & 4 & -63.4 & -0.4 \\
\hline$g$ & 6 & 5 & 14.7 & -0.4 \\
\hline$h$ & 6 & 5 & 0.0 & -0.2 \\
\hline$g$ & 6 & 6 & -86.4 & 1.3 \\
\hline$h$ & 6 & 6 & 50.3 & 0.9 \\
\hline$g$ & 7 & 0 & 79.8 & -0.4 \\
\hline$g$ & 7 & 1 & -74.4 & 0.0 \\
\hline$h$ & 7 & 1 & -61.4 & 0.8 \\
\hline$g$ & 7 & 2 & -1.4 & -0.2 \\
\hline$h$ & 7 & 2 & -22.5 & 0.4 \\
\hline$g$ & 7 & 3 & 38.6 & 1.1 \\
\hline$h$ & 7 & 3 & 6.9 & 0.1 \\
\hline$g$ & 7 & 4 & 12.3 & 0.6 \\
\hline$h$ & 7 & 4 & 25.4 & 0.2 \\
\hline$g$ & 7 & 5 & 9.4 & 0.4 \\
\hline$h$ & 7 & 5 & 10.9 & -0.9 \\
\hline$g$ & 7 & 6 & 5.5 & -0.5 \\
\hline$h$ & 7 & 6 & -26.4 & -0.3 \\
\hline$g$ & 7 & 7 & 2.0 & 0.9 \\
\hline$h$ & 7 & 7 & -4.8 & 0.3 \\
\hline$g$ & 8 & 0 & 24.8 & -0.2 \\
\hline$g$ & 8 & 1 & 7.7 & 0.2 \\
\hline
\end{tabular}

Table 1. (continued).

\begin{tabular}{|c|c|c|c|c|}
\hline$g / h$ & $n$ & $m$ & $\begin{array}{l}\text { MF } 2005 \\
\text { (nT) }\end{array}$ & $\begin{array}{c}\text { SV 2005-2010 } \\
\text { (nT/year) }\end{array}$ \\
\hline$h$ & 8 & 1 & 11.2 & -0.2 \\
\hline$g$ & 8 & 2 & -11.4 & -0.2 \\
\hline$h$ & 8 & 2 & -21.0 & 0.2 \\
\hline$g$ & 8 & 3 & -6.8 & 0.2 \\
\hline$h$ & 8 & 3 & 9.7 & 0.2 \\
\hline$g$ & 8 & 4 & -18.0 & -0.2 \\
\hline$h$ & 8 & 4 & -19.8 & 0.4 \\
\hline$g$ & 8 & 5 & 10.0 & 0.2 \\
\hline$h$ & 8 & 5 & 16.1 & 0.2 \\
\hline$g$ & 8 & 6 & 9.4 & 0.5 \\
\hline$h$ & 8 & 6 & 7.7 & -0.3 \\
\hline$g$ & 8 & 7 & -11.4 & -0.7 \\
\hline$h$ & 8 & 7 & -12.8 & 0.5 \\
\hline$g$ & 8 & 8 & -5.0 & 0.5 \\
\hline$h$ & 8 & 8 & -0.1 & 0.4 \\
\hline$g$ & 9 & 0 & 5.6 & \\
\hline$g$ & 9 & 1 & 9.8 & \\
\hline$h$ & 9 & 1 & -20.1 & \\
\hline$g$ & 9 & 2 & 3.6 & \\
\hline$h$ & 9 & 2 & 12.9 & \\
\hline$g$ & 9 & 3 & -7.0 & \\
\hline$h$ & 9 & 3 & 12.7 & \\
\hline$g$ & 9 & 4 & 5.0 & \\
\hline$h$ & 9 & 4 & -6.7 & \\
\hline$g$ & 9 & 5 & -10.8 & \\
\hline$h$ & 9 & 5 & -8.1 & \\
\hline$g$ & 9 & 6 & -1.3 & \\
\hline$h$ & 9 & 6 & 8.1 & \\
\hline$g$ & 9 & 7 & 8.7 & \\
\hline$h$ & 9 & 7 & 2.9 & \\
\hline$g$ & 9 & 8 & -6.7 & \\
\hline$h$ & 9 & 8 & -7.9 & \\
\hline$g$ & 9 & 9 & -9.2 & \\
\hline$h$ & 9 & 9 & 5.9 & \\
\hline$g$ & 10 & 0 & -2.2 & \\
\hline$g$ & 10 & 1 & -6.3 & \\
\hline$h$ & 10 & 1 & 2.4 & \\
\hline$g$ & 10 & 2 & 1.6 & \\
\hline$h$ & 10 & 2 & 0.2 & \\
\hline$g$ & 10 & 3 & -2.5 & \\
\hline$h$ & 10 & 3 & 4.4 & \\
\hline$g$ & 10 & 4 & -0.1 & \\
\hline$h$ & 10 & 4 & 4.7 & \\
\hline$g$ & 10 & 5 & 3.0 & \\
\hline$h$ & 10 & 5 & -6.5 & \\
\hline$g$ & 10 & 6 & 0.3 & \\
\hline$h$ & 10 & 6 & -1.0 & \\
\hline$g$ & 10 & 7 & 2.1 & \\
\hline$h$ & 10 & 7 & -3.4 & \\
\hline$g$ & 10 & 8 & 3.9 & \\
\hline$h$ & 10 & 8 & -0.9 & \\
\hline$g$ & 10 & 9 & -0.1 & \\
\hline$h$ & 11 & 1 & 0.3 & \\
\hline$h$ & 10 & 9 & -2.3 & \\
\hline$g$ & 10 & 10 & -2.2 & \\
\hline$h$ & 10 & 10 & -8.0 & \\
\hline$g$ & 11 & 0 & 2.9 & \\
\hline$g$ & 11 & 1 & -1.6 & \\
\hline$g$ & 11 & 2 & -1.7 & \\
\hline$h$ & 11 & 2 & 1.4 & \\
\hline$g$ & 11 & 3 & 1.5 & \\
\hline$h$ & 11 & 3 & -0.7 & \\
\hline$g$ & 11 & 4 & -0.2 & \\
\hline$h$ & 11 & 4 & -2.4 & \\
\hline$g$ & 11 & 5 & 0.2 & \\
\hline$h$ & 11 & 5 & 0.9 & \\
\hline$g$ & 11 & 6 & -0.7 & \\
\hline
\end{tabular}


Table 1. (continued).

\begin{tabular}{|c|c|c|c|c|}
\hline$g / h$ & $n$ & $m$ & $\begin{array}{c}\text { MF } 2005 \\
(\mathrm{nT})\end{array}$ & $\begin{array}{c}\text { SV 2005-2010 } \\
\text { (nT/year) }\end{array}$ \\
\hline$h$ & 11 & 6 & -0.6 & \\
\hline$g$ & 11 & 7 & 0.5 & \\
\hline$h$ & 11 & 7 & -2.7 & \\
\hline$g$ & 11 & 8 & 1.8 & \\
\hline$h$ & 11 & 8 & -1.0 & \\
\hline$g$ & 11 & 9 & 0.1 & \\
\hline$h$ & 11 & 9 & -1.5 & \\
\hline$g$ & 11 & 10 & 1.0 & \\
\hline$h$ & 11 & 10 & -2.0 & \\
\hline$g$ & 11 & 11 & 4.1 & \\
\hline$h$ & 11 & 11 & -1.4 & \\
\hline$g$ & 12 & 0 & -2.2 & \\
\hline$g$ & 12 & 1 & -0.3 & \\
\hline$h$ & 12 & 1 & -0.5 & \\
\hline$g$ & 12 & 2 & 0.3 & \\
\hline$h$ & 12 & 2 & 0.3 & \\
\hline$g$ & 12 & 3 & 0.9 & \\
\hline$h$ & 12 & 3 & 2.3 & \\
\hline$g$ & 12 & 4 & -0.4 & \\
\hline$h$ & 12 & 4 & -2.7 & \\
\hline$g$ & 12 & 5 & 1.0 & \\
\hline$h$ & 12 & 5 & 0.6 & \\
\hline$g$ & 12 & 6 & -0.4 & \\
\hline$h$ & 12 & 6 & 0.4 & \\
\hline$g$ & 12 & 7 & 0.5 & \\
\hline$h$ & 12 & 7 & 0.0 & \\
\hline$g$ & 12 & 8 & -0.3 & \\
\hline$h$ & 12 & 8 & 0.0 & \\
\hline$g$ & 12 & 9 & -0.4 & \\
\hline$h$ & 12 & 9 & 0.3 & \\
\hline$g$ & 12 & 10 & 0.0 & \\
\hline$h$ & 12 & 10 & -0.8 & \\
\hline$g$ & 12 & 11 & -0.4 & \\
\hline$h$ & 12 & 11 & -0.4 & \\
\hline$g$ & 12 & 12 & 0.0 & \\
\hline$h$ & 12 & 12 & 1.0 & \\
\hline$g$ & 13 & 0 & -0.2 & \\
\hline$g$ & 13 & 1 & -0.9 & \\
\hline$h$ & 13 & 1 & -0.7 & \\
\hline$g$ & 13 & 2 & 0.3 & \\
\hline$h$ & 13 & 2 & 0.3 & \\
\hline$g$ & 13 & 3 & 0.3 & \\
\hline$h$ & 13 & 3 & 1.7 & \\
\hline$g$ & 13 & 4 & -0.4 & \\
\hline$h$ & 13 & 4 & -0.5 & \\
\hline$g$ & 13 & 5 & 1.2 & \\
\hline$h$ & 13 & 5 & -1.0 & \\
\hline$g$ & 13 & 6 & -0.4 & \\
\hline$h$ & 13 & 6 & 0.0 & \\
\hline$g$ & 13 & 7 & 0.7 & \\
\hline$h$ & 13 & 7 & 0.7 & \\
\hline$g$ & 13 & 8 & -0.3 & \\
\hline$h$ & 13 & 8 & 0.2 & \\
\hline$g$ & 13 & 9 & 0.4 & \\
\hline$h$ & 13 & 9 & 0.6 & \\
\hline$g$ & 13 & 10 & -0.1 & \\
\hline$h$ & 13 & 10 & 0.4 & \\
\hline$g$ & 13 & 11 & 0.4 & \\
\hline$h$ & 13 & 11 & -0.2 & \\
\hline$g$ & 13 & 12 & -0.1 & \\
\hline$h$ & 13 & 12 & -0.5 & \\
\hline$g$ & 13 & 13 & -0.3 & \\
\hline$h$ & 13 & 13 & -1.0 & \\
\hline
\end{tabular}
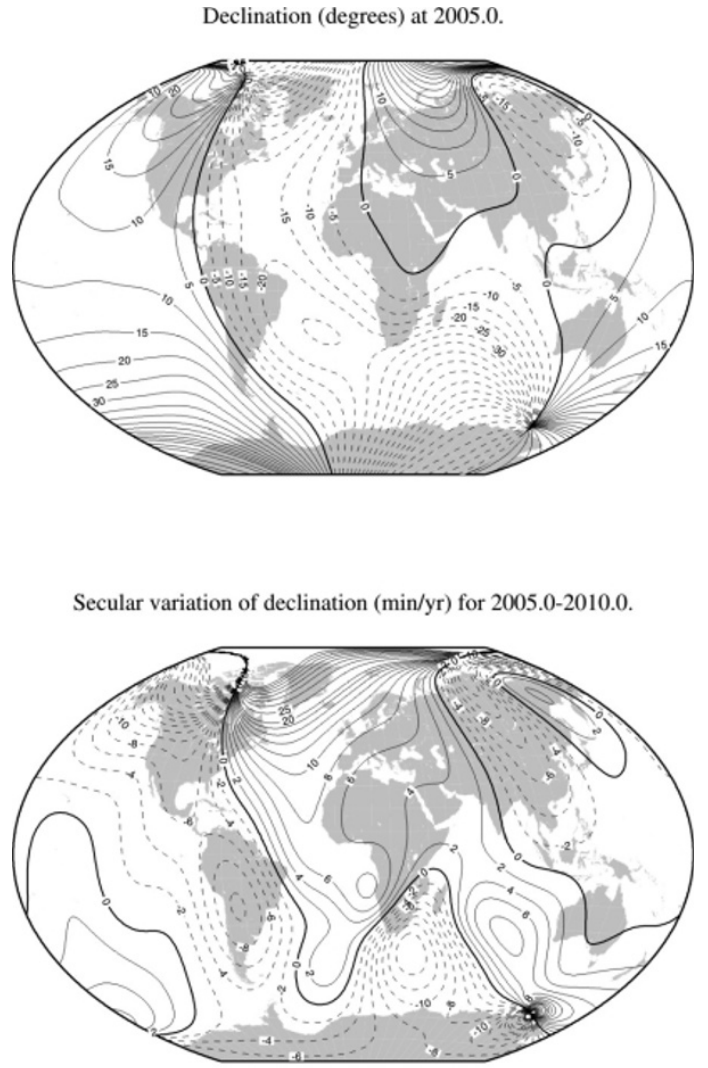

Projection: Winkel Tripel

Fig. 1. Contour maps for the D component from IGRF-10.

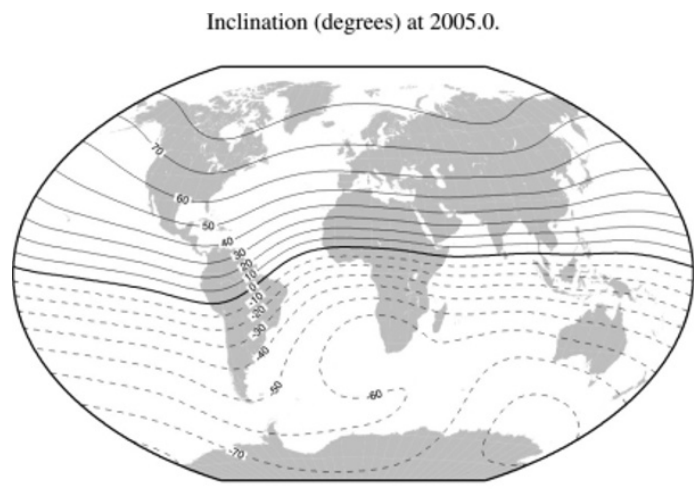

Secular variation of inclination $(\mathrm{min} / \mathrm{yr})$ for 2005.0-2010.0.

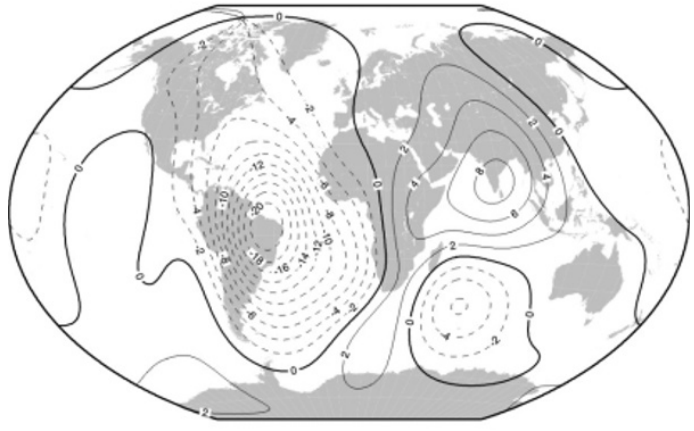

Projection: Winkel Tripel

Fig. 2. Contour maps for the I component from IGRF-10. 


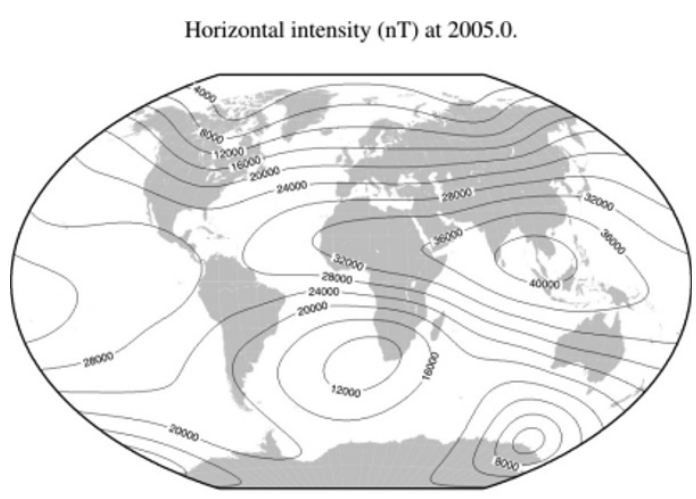

Secular variation of horizontal intensity (nT/yr) for 2005.0-2010.0.

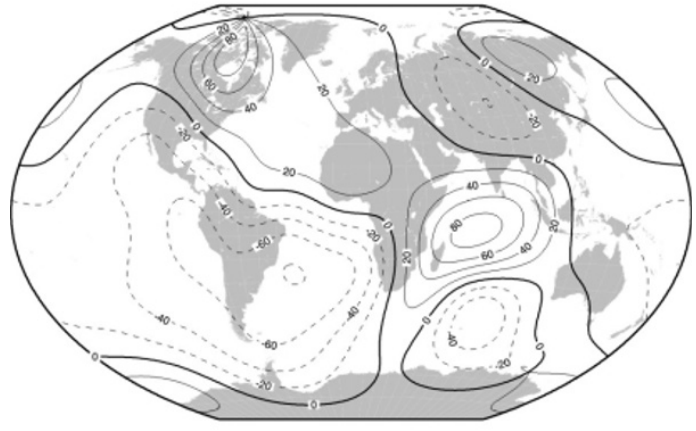

Projection: Winkel Tripel

Fig. 3. Contour maps of the H component from IGRF-10.

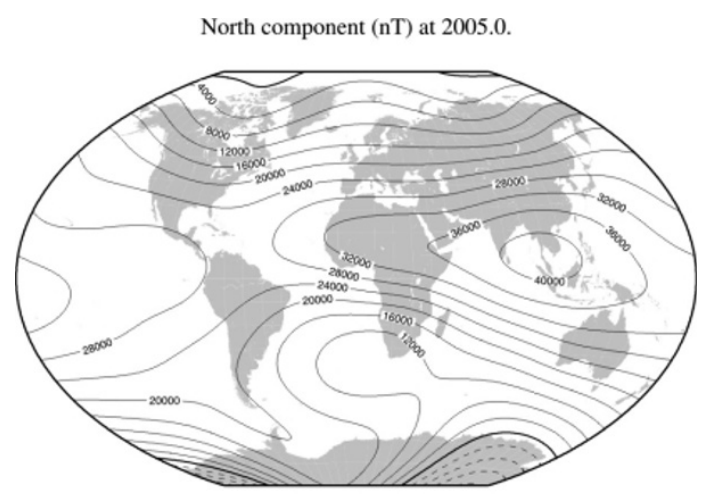

Secular variation of north component (nT/yr) for 2005.0-2010.0.

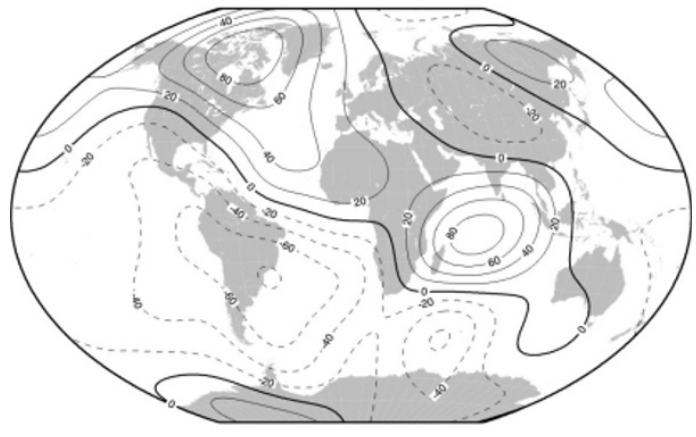

Projection: Winkel Tripel

Fig. 4. Contour maps for the X component from IGRF-10.

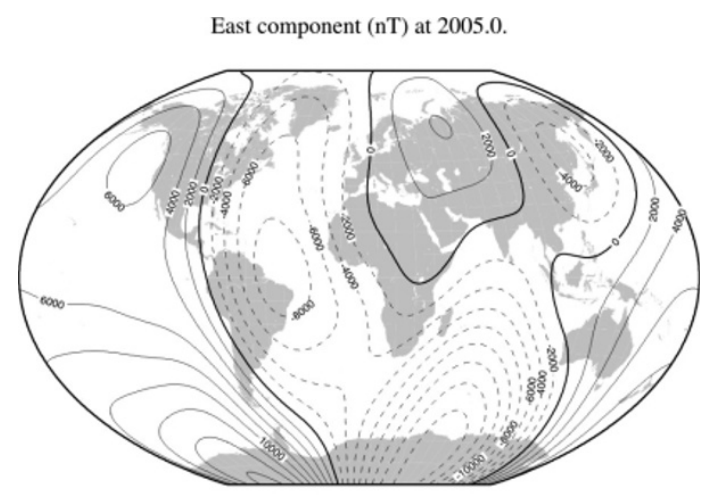

Secular variation of east component (nT/yr) for 2005.0-2010.0.

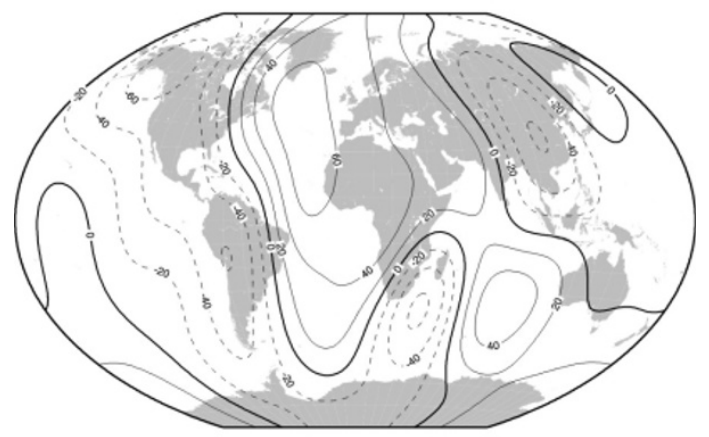

Projection: Winkel Tripel

Fig. 5. Contour maps for the Y component from IGRF-10.

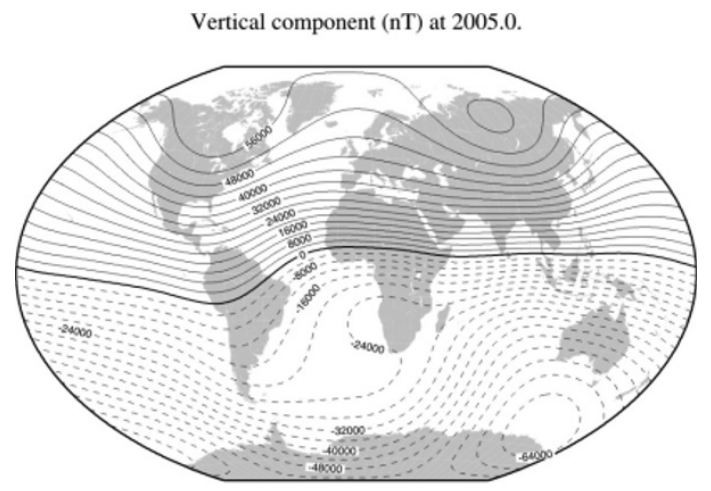

Secular variation of vertical component (nT/yr) for 2005.0-2010.0.

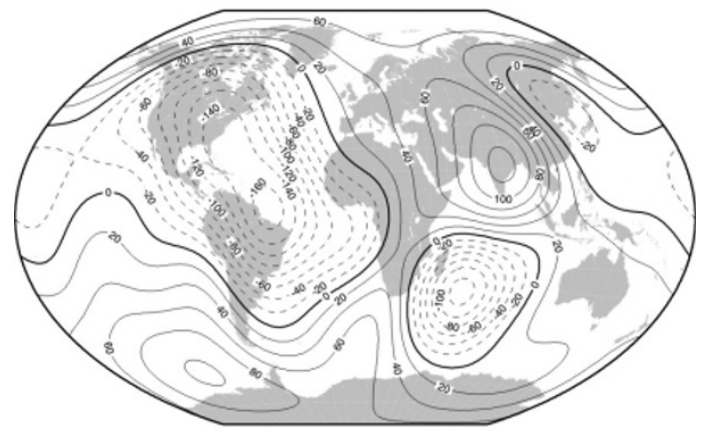

Projection: Winkel Tripel

Fig. 6. Contour maps for the $\mathrm{Z}$ component from IGRF-10. 

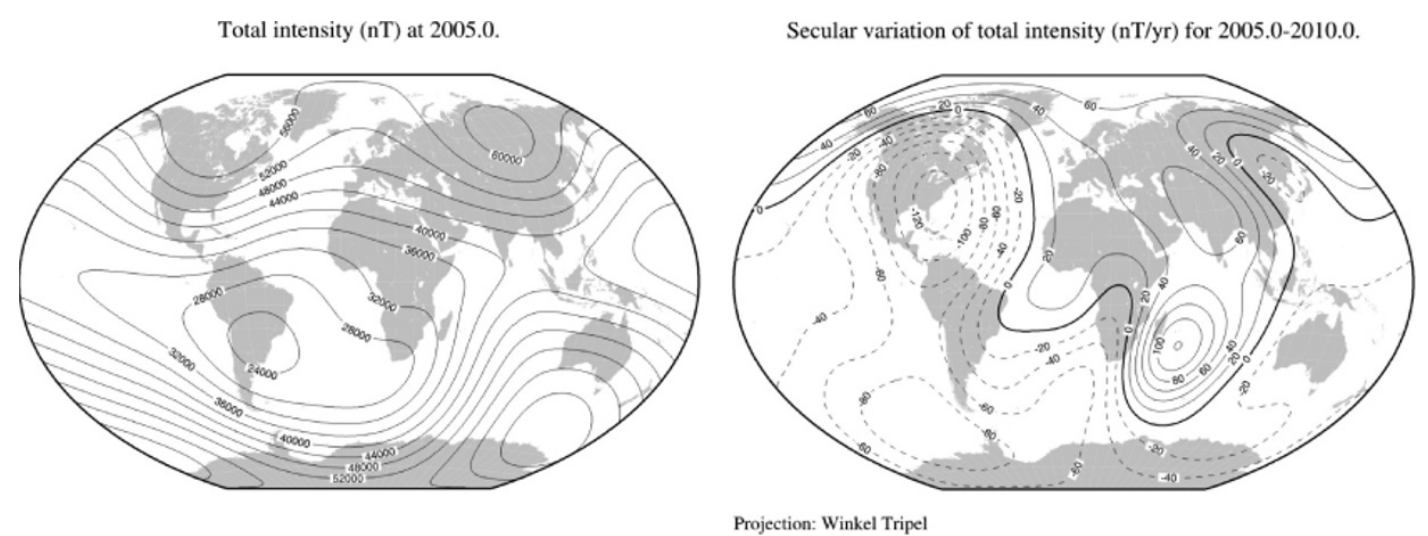

Fig. 7. Contour maps of the F component from IGRF-10.

Table 2. Summary of nomenclature and IGRF history.

\begin{tabular}{lccc}
\hline Full name & Short name & Valid for & Definitive for \\
\hline IGRF 10th generation (revised 2004) & IGRF-10 & $1900.0-2010.0$ & $1945.0-2000.0$ \\
IGRF 9th generation (revised 2003) & IGRF-9 & $1900.0-2005.0$ & $1945.0-2000.0$ \\
IGRF 8th generation (revised 1999) & IGRF-8 & $1900.0-2005.0$ & $1945.0-1990.0$ \\
IGRF 7th generation (revised 1995) & IGRF-7 & $1900.0-2000.0$ & $1945.0-1990.0$ \\
IGRF 6th generation (revised 1991) & IGRF-6 & $1945.0-1995.0$ & $1945.0-1985.0$ \\
IGRF 5th generation (revised 1987) & IGRF-5 & $1945.0-1990.0$ & $1945.0-1980.0$ \\
IGRF 4th generation (revised 1985) & IGRF-4 & $1945.0-1990.0$ & $1965.0-1980.0$ \\
IGRF 3rd generation (revised 1981) & IGRF-3 & $1965.0-1985.0$ & $1965.0-1975.0$ \\
IGRF 2nd generation (revised 1975) & IGRF-2 & $1955.0-1980.0$ & - \\
IGRF 1st generation (revised 1969) & IGRF-1 & $1955.0-1975.0$ & - \\
\hline
\end{tabular}

candidate models was taken at the start of December 2004. Thus the new coefficients extending the previous generation of the IGRF to beyond its expiry date at end of 2004 were available on the internet in time.

\section{Coefficients and Maps}

The coefficients of the main-field model at 2005.0 and secular-variation model for 2005.0-2010.0 are listed in Table 1 and are available in digital form from the IAGA web site www.iugg.org/IAGA and the World Data Centres listed at the end of this paper, along with software to compute magnetic field values from them.

Figures 1-7 show global maps of the various magnetic elements and their annual rates of change for the period 2005.0-2010.0.

Table 2 gives the nomenclature that should be used with the IGRF, and gives a brief summary of its history (Barton, 1997).

It is recommended not to use the term IGRF without reference to the generation, as then it is difficult to establish which coefficients were actually used. For example, one cannot recover the original full-field data from an aeromagnetic anomaly dataset in order to tie it with adjacent surveys if one does not know which generation of the IGRF was used. It is also recommended that the full name be used, so that it is more apparent whether the output values are "predictive" and are therefore less accurate.

The World Geodetic System 1984 datum and spheroid is recommended for use in coordinate transformations as they are widely recognised as standards (major axis $=6378.137$ $\mathrm{km}$, minor axis $=6356.752 \mathrm{~km}$ ). Present-day satellite mag- netic data are mostly positioned using WGS84 but for other data we are often unaware which datum is used. Differences in output IGRF magnetic field values at the Earth's surface are less than $1 \mathrm{nT}$ when this spheroid is used in place of the former preferred spheroid, the International Astronomical Union 1966 spheroid.

Table 3. Positions of geomagnetic and magnetic poles 1900.0-2010.0 estimated from IGRF-10.

\begin{tabular}{l|cc|cc|cc}
\hline & \multicolumn{2}{|c|}{ North dipole pole } & \multicolumn{2}{|c|}{ North dip pole } & \multicolumn{2}{c}{ South dip pole } \\
\hline Epoch & Latitude Longitude & Latitude Longitude & Latitude Longitude \\
\hline 1900.0 & 78.68 & -68.79 & 70.46 & -96.19 & -71.72 & 148.32 \\
1905.0 & 78.68 & -68.75 & 70.66 & -96.48 & -71.46 & 148.55 \\
1910.0 & 78.66 & -68.72 & 70.79 & -96.72 & -71.15 & 148.64 \\
1915.0 & 78.64 & -68.57 & 71.03 & -97.03 & -70.80 & 148.54 \\
1920.0 & 78.63 & -68.38 & 71.34 & -97.39 & -70.41 & 148.20 \\
1925.0 & 78.62 & -68.27 & 71.79 & -98.00 & -69.99 & 147.63 \\
1930.0 & 78.60 & -68.26 & 72.27 & -98.69 & -69.52 & 146.79 \\
1935.0 & 78.57 & -68.36 & 72.80 & -99.34 & -69.06 & 145.77 \\
1940.0 & 78.55 & -68.51 & 73.30 & -99.87 & -68.57 & 144.60 \\
1945.0 & 78.55 & -68.53 & 73.93 & -100.24 & -68.15 & 144.44 \\
1950.0 & 78.55 & -68.85 & 74.64 & -100.86 & -67.89 & 143.55 \\
1955.0 & 78.54 & -69.16 & 75.18 & -101.41 & -67.19 & 141.50 \\
1960.0 & 78.58 & -69.47 & 75.30 & -101.03 & -66.70 & 140.23 \\
1965.0 & 78.60 & -69.85 & 75.63 & -101.34 & -66.33 & 139.53 \\
1970.0 & 78.66 & -70.18 & 75.88 & -100.98 & -66.02 & 139.40 \\
1975.0 & 78.76 & -70.47 & 76.15 & -100.64 & -65.74 & 139.52 \\
1980.0 & 78.88 & -70.76 & 76.91 & -101.68 & -65.42 & 139.34 \\
1985.0 & 79.04 & -70.90 & 77.40 & -102.61 & -65.13 & 139.18 \\
1990.0 & 79.21 & -71.13 & 78.09 & -103.68 & -64.91 & 138.90 \\
1995.0 & 79.39 & -71.42 & 79.09 & -105.42 & -64.79 & 138.76 \\
2000.0 & 79.61 & -71.57 & 80.97 & -109.64 & -64.66 & 138.30 \\
2005.0 & 79.81 & -71.78 & 83.23 & -118.31 & -64.54 & 137.86 \\
2010.0 & 80.02 & -71.98 & 85.19 & -133.16 & -64.44 & 137.44 \\
\hline & & & & & &
\end{tabular}




\section{Pole Positions Computed from IGRF-10}

One use of the IGRF is for computing locations of poles through time. Table 3 lists the locations of the geomagnetic (or dipole) north pole (the south pole is exactly antipodal to this) and the magnetic (or dip) poles. These have been computed at 5-year intervals using the 10th generation IGRF.

\section{World Data Centres}

WDC for Solid Earth Geophysics, National Geophysical Data Center, 325 Broadway, Boulder, CO 80303-3328, USA

Email: Susan.McLean@noaa.gov

Internet: www.ngdc.noaa.gov

WDC for Geomagnetism, Data Analysis Center for Geomagnetism and Space Magnetism, Graduate School of Science, Kyoto University, Kyoto 606-8502, JAPAN

Email: iyemori@kugi.kyoto-u.ac.jp

Internet: swdcwww.kugi.kyoto-u.ac.jp

WDC for Geomagnetism, British Geological Survey, Murchison House, West Mains Road, Edinburgh, EH9 3LA, UK

Email:smac@bgs.ac.uk

Internet: www.geomag.bgs.ac.uk

Table 4. List of agencies supporting observatories whose data were used in deriving the new constituent models in IGRF-10.

\begin{tabular}{|c|c|c|}
\hline $\begin{array}{l}\text { Supporting Agency } \\
\end{array}$ & Country & Observatory IAGA code \\
\hline $\begin{array}{l}\text { Centre de Recherche en Astronomie } \\
\text { Astrophysique et Geophysique }\end{array}$ & ALGERIA & TAM \\
\hline Servicio Meteorologico Nacional & ARGENTINA & PIL \\
\hline Universidad Nacional de la Plata & ARGENTINA & LAS, TRW \\
\hline Geoscience Australia & AUSTRALIA & $\begin{array}{l}\text { ASP, CNB, CSY, CTA, DVS, GNA, KDU, } \\
\text { LRM, MAW, MCQ }\end{array}$ \\
\hline $\begin{array}{l}\text { Zentralanstalt für Meteorologie und } \\
\text { Geodynamik }\end{array}$ & AUSTRIA & WIK \\
\hline Institut Royal Météorologique & BELGIUM & DOU, MAB \\
\hline CNPq-Observatório Nacional & BRAZIL & TTB, VSS \\
\hline Academy of Sciences & BULGARIA & PAG \\
\hline Geological Survey of Canada & CANADA & $\begin{array}{l}\text { ALE, BLC, CBB, FCC, GLN, IQA, MBC, } \\
\text { MEA, OTT, PBQ, RES, STJ, VIC,YKC }\end{array}$ \\
\hline Academy of Sciences & CHINA & BMT \\
\hline State Seismological Bureau & CHINA & $\begin{array}{l}\text { BJI, CDP, CHD, CNH, DLN, GLM, GZH, } \\
\text { KSH, LSA, LZH, MZL, QGZ, QIX, QZH, } \\
\text { SSH, THJ, WHN, WMQ }\end{array}$ \\
\hline Instituto Geográfico Agustín Codazzi & COLOMBIA & FUQ \\
\hline Instituto Costarricense de Electricidad & COSTA RICA & CRP \\
\hline Academy of Sciences & CZECH REPUBLIC & BDV \\
\hline Danish Meteorological Institute & \begin{tabular}{|l} 
DENMARK \\
\end{tabular} & BFE, GDH, NAQ, THL \\
\hline $\begin{array}{l}\text { National Research Institute of Astronomy and } \\
\text { Geophysics }\end{array}$ & EGYPT & \begin{tabular}{|l|l} 
MLT \\
\end{tabular} \\
\hline Addis Ababa University & ETHIOPIA & AAE \\
\hline Finnish Meteorological Institute & FINLAND & NUR \\
\hline Geophysical Observatory & FINLAND & OUL, SOD \\
\hline Institut de Physique du Globe de Paris & FRANCE & CLF, KOU, PPT \\
\hline Ecole et Observatoire des Sciences de la Terre & FRANCE & AMS, CZT, DRV, PAF \\
\hline $\begin{array}{l}\text { Institut Français de Recherche Scientifique } \\
\text { pour le Développement }\end{array}$ & FRANCE & BNG, MBO \\
\hline Academy of Sciences & GEORGIA & TFS \\
\hline Universitat München & GERMANY & FUR \\
\hline $\begin{array}{l}\text { Alfred-Wegener-Institute for Polar \& } \\
\text { Marine Research }\end{array}$ & GERMANY & VNA \\
\hline GeoForschungsZentrum Potsdam & GERMANY & NGK, WNG \\
\hline Institute of Geology and Mineral Exploration & GREECE & PEG \\
\hline Academy of Sciences & HUNGARY & NCK \\
\hline Eötvös Loránd Geophysical Institute & HUNGARY & THY \\
\hline University of Iceland & ICELAND & LRV \\
\hline Indian Institute of Geomagnetism & INDIA & $\begin{array}{l}\text { ABG, NGP, PND, SHL, SIL, TIR, TRD, } \\
\text { UJJ, VSK }\end{array}$ \\
\hline National Geophysical Research Institute & INDIA & ETT, HYB \\
\hline Survey of India & INDIA & SAB \\
\hline Badan Meteorologi dan Geofisika & \begin{tabular}{|l|} 
INDONESIA \\
\end{tabular} & \begin{tabular}{|l|} 
TND, TUN \\
\end{tabular} \\
\hline Meteorological and Geophysical Agency & INDONESIA & TNG \\
\hline Meteorological Service & IRELAND & VAL \\
\hline Survey of Israel & ISRAEL & AMT, BGY, ELT \\
\hline Instituto Nazionale di Geofisica & ITALY & AQU, CTS, TNB \\
\hline Japan Coast Guard & JAPAN & HTY \\
\hline Japan Meteorological Agency & JAPAN & CBI, KAK, KNY, MMB \\
\hline Geographical Survey Institute & JAPAN & ESA, KNZ, MIZ \\
\hline
\end{tabular}

Table 4. (continued)

\begin{tabular}{|c|c|c|}
\hline Supporting Agency & Country & Observatory IAGA code \\
\hline National Institute of Polar Research & JAPAN & SYO \\
\hline Ministry of Education and Science & KAZAKHSTAN & AAA \\
\hline National Centre for Geophysical Research & \begin{tabular}{|l|l|} 
LEBANON \\
\end{tabular} & QSB \\
\hline Université d'Antananarivo & MADAGASCAR & TAN \\
\hline Ciudad Universitaria & MEXICO & TEO \\
\hline Institute of Geological and Nuclear Sciences & NEW ZEALAND & API, EYR, SBA \\
\hline University of Bergen & NORWAY & DOB \\
\hline University of Troms $\emptyset$ & NORWAY & BJN, DOB, NAL, TRO \\
\hline $\begin{array}{l}\text { Space \& Upper Atmosphere Research } \\
\text { Commission }\end{array}$ & PAKISTAN & KRC \\
\hline Meteorological Department & PAKISTAN & QUE \\
\hline Instituto Geofisico del Peru & PERU & ANC, HUA \\
\hline Academy of Sciences & POLAND & BEL, HLP, HRN \\
\hline Universidade de Coimbra & PORTUGAL & COI \\
\hline Directorate General of Telecommunications & $\begin{array}{l}\text { REPUBLIC OF } \\
\text { CHINA }\end{array}$ & LNP \\
\hline Instituto Nacional de Geologia & $\begin{array}{l}\text { REPÚBLICA DE } \\
\text { MOÇAMBIQUE }\end{array}$ & LMM \\
\hline Geological Survey of Romania & ROMANIA & SUA \\
\hline Arctic and Antarctic Research Institute & RUSSIA & CCS, DIK, HIS, MIR, MOL, TIK, VOS \\
\hline Academy of Sciences & RUSSIA & $\begin{array}{l}\text { ARS, ASH, BOX, KIV, KZN, LNN, LVV, } \\
\text { MGD, MNK, MOS, NKK, NVS, ODE, } \\
\text { PET, POD, TKT, VLA, YAK }\end{array}$ \\
\hline Institute of Solar-Terrestrial Physics & RUSSIA & IRT \\
\hline Slovenska Akademia Vied & SLOVAKIA & HRB \\
\hline National Research Foundation & SOUTH AFRICA & HBK, HER, TSU \\
\hline Observatori de l'Ebre & SPAIN & EBR, LIV \\
\hline Real Instituto y Observatorio de la Armada & SPAIN & SFS \\
\hline Instituto Geográfico Nacional & SPAIN & GUI, SPT \\
\hline Sveriges Geologiska Undersökning & SWEDEN & ABK, LOV, UPS \\
\hline Swedish Institute of Space Physics & SWEDEN & KIR \\
\hline Boğaziçi University & TURKEY & ISK \\
\hline Academy of Sciences & \begin{tabular}{|l} 
UKRAINE \\
\end{tabular} & AIA \\
\hline British Geological Survey & UNITED KINGDOM & ASC, ESK, HAD, LER, PST \\
\hline US Geological Survey & \begin{tabular}{|l} 
UNITED STATES \\
\end{tabular} & $\begin{array}{l}\text { BRW, BOU, BSL, CMO, DLR, FRD, FRN, } \\
\text { GUA, HON, MID, NEW, SIT, SJG, TUC }\end{array}$ \\
\hline National Centre for Science and Technology & VIETNAM & CPA, PHU \\
\hline Geomagnetic Institute & YUGOSLAVIA & GCK \\
\hline
\end{tabular}

Acknowledgments. The home institutes of the various authors of papers in this special issue and the many organisations involved in operating magnetic survey satellites, observatories (see Table 4 prepared with assistance from Jeff Love and Jill Caldwell, USGS), magnetic survey programmes and World Data Centres are to be thanked for their continuing support of the IGRF project.

\section{References}

Barton, C. E., International Geomagnetic Reference Field: the seventh generation, J. Geomag. Geoelectr., 49, 123-148, 1997.

Chapman, S. and J. Bartels, Geomagnetism (2 Vols) Oxford University Press, London, 1940.

Golovkov, V. P., T. I. Zvereva, and T. A. Chernova, The IZMIRAN main magnetic field candidate model for IGRF-10, produced by a spherical harmonic-Natural orthogonal component method, Earth Planets Space, 57, this issue, 1165-1171, 2005.

Langel, R. A., Main Field, in Geomagnetism, Vol. 1, edited by J. A. Jacobs, Academic Press, London, 1987.

Lesur, V., S. Macmillan, and A. Thomson, The BGS magnetic field candidate models for the 10th generation IGRF, Earth Planets Space, 57, this issue, $1157-1163,2005$.

Maus, S., S. Macmillan, F. Lowes, and T. Bondar, Evaluation of candidate geomagnetic field models for the 10th generation of IGRF, Earth Planets Space, 57, this issue, 1173-1181, 2005a.

Maus, S., S. McLean, D. Dater, H. Lühr, M. Rother, W. Mai, and S. Choi, NGDC/GFZ candidate models for the 10th generation International Geomagnetic Reference Field, Earth Planets Space, 57, this issue, 1151$1156,2005 b$.

Olsen, N., T. Sabaka, and F. Lowes, New plarameterization of external and induced fields in geomagnetic field modeling, and a candidate model for IGRF 2005, Earth Planets Space, 57, this issue, 1141-1149, 2005.

S. Macmillan (e-mail: smac@bgs.ac.uk) and S. Maus 